\title{
Evidence for a different type of vortex that mediates a continuous fluxoid-state transition in a mesoscopic superconducting ring
}

\author{
A. Kanda, ${ }^{1}$ B. J. Baelus, ${ }^{2}$ D. Y. Vodolazov,${ }^{2,3}$ J. Berger, ${ }^{4}$ R. Furugen,${ }^{1}$ Y. Ootuka,${ }^{1}$ and F. M. Peeters ${ }^{2}$ \\ ${ }^{1}$ Institute of Physics and TIMS, University of Tsukuba, Tsukuba 305-8571, Japan \\ ${ }^{2}$ Departement Fysica, Universiteit Antwerpen, Groenenborgerlaan 171, B-2020 Antwerpen, Belgium \\ ${ }^{3}$ Institute for Physics of Microstructures, Russian Academy of Sciences, 603950, Nizhny Novgorod GSP-105, Russia \\ ${ }^{4}$ Department of Physics, Ort-Braude College, P.O. Box 78, 21982 Karmiel, Israel
}

(Received 8 June 2007; published 26 September 2007)

\begin{abstract}
The magnetic field response of a mesoscopic superconducting ring with an inside hole positioned off-center is studied by using the multiple-small-tunnel-junction method, by which the strengths of the superconductivity at the narrowest and the widest parts of the ring are detected separately and simultaneously. We observed continuous and reversible transitions between adjacent fluxoid states at temperatures close to the onset of superconductivity. Our findings are in agreement with numerical ones based on the Ginzburg-Landau theory, and show that in such continuous transitions, a different type of vortex nucleates at the narrowest part of the ring in the case of a low flux and at the widest part for a larger flux. The former corresponds to a onedimensional vortex, which has been predicted theoretically for more than a decade.
\end{abstract}

DOI: 10.1103/PhysRevB.76.094519

PACS number(s): 74.78.Na, 74.25.Dw, 74.25.Op

\section{INTRODUCTION}

In mesoscopic superconductors, which have sizes comparable to the superconducting coherence length $\xi$ or the magnetic penetration depth $\lambda$, the quantum confinement effect dramatically modifies the superconducting properties. Recent development of nanofabrication techniques as well as progress in the numerical simulation methods allow one to investigate such new quantum effects both experimentally and theoretically. For example, in mesoscopic thin films, the confinement leads to the formation of novel vortex states such as giant vortex states, multivortex states, ${ }^{1-9}$ and vortexantivortex molecules. ${ }^{10-14}$ Some of them have been confirmed experimentally. ${ }^{15-21}$ For mesoscopic rings, theories have predicted that the transition between different fluxoid states may lead to reversible and continuous variation of related physical quantities, such as magnetization, supercurrent, and superconducting order parameter, when the cross section of the ring varies along the circumference. This is associated with the nucleation of a novel type of vortices. ${ }^{22,23}$ Note that either in bulk hollow cylinders (rings) or in films of type-II superconductors, transitions between quantum states with different fluxoid quantum numbers (or vorticities) are discontinuous and irreversible. In this paper, we focus on the vortex state which mediates reversible and continuous fluxoid-state transitions in an asymmetric ring.

The unconventional behavior in mesoscopic rings was first derived in the one-dimensional (1D) limit. ${ }^{24-30}$ In the 1980 s, it was found that in a 1D ring with an attached lead, a magnetic flux $\Phi$ induces a nonuniform distribution of the order parameter, and the order parameter vanishes at one point of the ring when $\Phi=\Phi_{0} / 2\left(\Phi_{0}\right.$ is the magnetic flux quantum). ${ }^{24-26}$ Later, Berger and Rubinstein ${ }^{27,29}$ found that in the 1D limit in a ring with nonuniform cross section, there is a stable superconducting state at the onset of superconductivity in which a part of the sample is normal, leading to a singly connected superconducting state. At a lower temperature, there is a critical point at which the magnetic suscepti- bility diverges. Some of $\mathrm{us}^{23}$ pointed out that the phase of the superconducting order parameter jumps by $\pi$ at the point where the order parameter vanishes, having the property of a vortex. They called it a $1 \mathrm{D}$ vortex. The analyses were extended to rings with finite and nonuniform cross section, allowing the variation of the order parameter in the radial direction, and the properties corresponding to the $1 \mathrm{D}$ case were obtained as described below. ${ }^{22,23}$

The present vortex is predicted to have the following properties: ${ }^{22,23}$ (i) This vortex is stabilized only in mesoscopic superconducting rings having diameter smaller than the coherence length $\xi$ and nonuniform cross section. Its existence is irrespective of the type of superconductor (type I or II). (ii) Analogous to the Abrikosov vortices in type-II superconductors, the superconducting phase changes by $2 \pi$ when encircling the core. Besides, the present vortex can be located even at the sample boundary. In this case, the vortex can be identified by the phase change of $\pi$ when going through the core. This is in contrast to the Abrikosov vortex that is unstable when the distance to the boundary is too short. Furthermore, the present vortex can be stabilized in a thin ring with width smaller than $\xi$, where the vortex becomes highly anisotropic and the region where the order parameter $\Psi$ is close to zero extends over the whole width. This vortex corresponds to a $1 \mathrm{D}$ vortex derived in the $1 \mathrm{D}$ limit. ${ }^{23}$ (iii) The vortex exists only in a narrow range of applied magnetic flux in the vicinity of a half-integer number of flux quanta: as the applied flux is increased (decreased), a vortex core appears at the outer (inner) edge of the ring, moves continuously toward the inner (outer) edge, and disappears at the inner (outer) edge. Thus, this vortex, also nicknamed a flux-induced vortex in Ref. 22, exists as a stable intermediate state during a transition between different fluxoid states. This fluxoid-state transition is reversible and continuous, in contrast to the usual transitions in rings which exhibit clear hysteresis and a jump in the current circulating around the loop. ${ }^{31}$ Besides, the vortex differs from the usual Abrikosov vortex in a film: in the latter case, when a vortex 


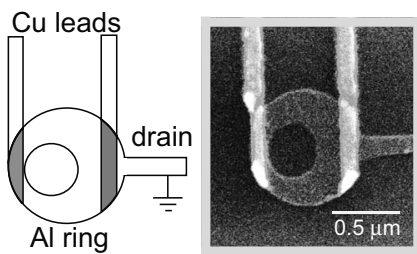

FIG. 1. A schematic view (left) and a scanning electron micrograph (right) of the sample. Two $\mathrm{Cu}$ leads are connected to an $\mathrm{Al}$ asymmetric ring through highly resistive small tunnel junctions (shaded areas). An Al drain is directly connected to the widest part of the ring. This structure was fabricated using $e$-beam lithography followed by double-angle evaporation of $\mathrm{Al}$ and $\mathrm{Cu}$. After the $\mathrm{Al}$ film was deposited, its surface was slightly oxidized to provide a tunnel barrier.

is nucleated at the sample edge, it inevitably jumps deep inside the sample at the same magnetic field, and it does not disappear at higher magnetic fluxes. (iv) For a mesoscopic ring with an off-centered hole, the vortex passes through the narrowest part of the ring for small magnetic fluxes, and through the widest part for large fluxes.

Although up to now several experimental studies were performed on mesoscopic rings and some of them observed a continuous transition between fluxoid states, there has been no clear experimental confirmation of this vortex. ${ }^{32-38}$ In the present paper, we experimentally demonstrate the penetration of vortices in asymmetric rings as a stable intermediate state in fluxoid-state transitions. Our results are confirmed with theoretical calculations based on the Ginzburg-Landau (GL) theory.

The paper is organized as follows. In Sec. II, we describe the experimental setup. In Sec. III, we present the theoretical formalisms used to obtain the numerical results. The experimental results together with the theoretical ones are described in Sec. IV. In Sec. V, we discuss the properties of the vortex state found in the present experiment in more detail. Finally, in Sec. VI, we summarize our results.

\section{EXPERIMENT}

To experimentally detect the properties of vortices expected for nonuniform mesoscopic rings, we probed the local strength of superconductivity. Here, we used the multiplesmall-tunnel-junction (MSTJ) method, ${ }^{18}$ in which several small tunnel junctions with high tunnel resistance are attached to a mesoscopic superconductor to simultaneously detect small changes in the local density of states (LDOS) under the junctions. Since the LDOS depends on the local superconducting energy gap $\Delta$, and hence the Cooper-pair density $|\Psi|^{2}$, the MSTJ method allows us to compare the strength of superconductivity under the different junctions.

Figure 1 shows a schematic view and a scanning electron microscopy image of the sample. Two normal-metal $(\mathrm{Cu})$ leads cover the narrowest and the widest parts of a superconducting $\mathrm{Al}$ ring with an off-centered hole (outer radius $r_{o}$ $=0.42 \mu \mathrm{m}$, inner radius $r_{i}=0.18 \mu \mathrm{m}$, displacement of the inner center $a=0.10 \mu \mathrm{m}$, and thickness $d=30 \mathrm{~nm}$ ), forming small $\mathrm{Al} / \mathrm{AlO}_{x} / \mathrm{Cu}$ tunnel junctions (shaded areas) with nominal tunnel resistance of several tens of kilohms. The ring is connected to an $\mathrm{Al}$ drain lead at the widest part. The sample was fabricated using $e$-beam lithography followed by double-angle evaporation of $\mathrm{Al}$ and $\mathrm{Cu}$ in a chamber with a base pressure of $2 \times 10^{-8} \mathrm{~Pa}$. The superconducting coherence length $\xi(T=0)$ was estimated to be $0.15 \pm 0.04 \mu \mathrm{m}$ from the residual resistance of $\mathrm{Al}$ films prepared in the same way. The superconducting transition temperature at zero magnetic field was $T_{c}=1.36 \mathrm{~K}$.

The sample was cooled in a dilution refrigerator that was equipped with low pass noise filters for measurement lines in the lowest temperature part. In the measurement, we connected a current source to each $\mathrm{Cu}$ lead and measured the current-voltage characteristics of the two junctions simultaneously as functions of the perpendicular magnetic field and the temperature. The sweep rate of the magnetic field was $3 \mathrm{mT} / \mathrm{min}$.

\section{THEORETICAL FORMALISM}

The numerical study of the experimental results was carried out using the two kinds of theoretical formalism used in Refs. 22 and 23.

\section{A. Perturbation method}

At the onset of superconductivity, the GL equation for the electromagnetic potential becomes irrelevant and the GL equation for the order parameter becomes a linear eigenvalue equation. Moreover, if there is cylindrical symmetry, the dependence on the coordinates $(r, \theta)$ can be separated and the possible solutions for the order parameter are

$$
\Psi^{(L)}(r, \theta)=\mathcal{R}_{L}(r) e^{-i L \theta},
$$

where $L$ is an integer. The functions $\mathcal{R}_{L}(r)$ and the temperatures for the onset of superconductivity can be evaluated analytically ${ }^{39}$ The most interesting situation is that in which $\Psi^{(L)}$ and $\Psi^{(L+1)}$ have the same eigenvalue. Let us denote the temperature and field at which this happens by $T_{L}$ and $H_{L}$, respectively.

Much can be learned by extending perturbatively the analytic results to situations in which the temperature is near the onset of superconductivity and the sample is close to cylindrical symmetry. In our experiment, low temperatures were reached and the sample was very eccentric; therefore, the perturbative approach gives just a crude approximation and the numerical method of the following section will be required. Nevertheless, the qualitative features predicted by the perturbative approach remain valid.

The perturbative treatment was performed in Ref. 40. In the following, we review those results that we will use for comparison with our experimental data. We define an eccentricity parameter

$$
\beta=2 \int_{0}^{2 \pi} w(\theta) \cos (\theta) d \theta / \int_{0}^{2 \pi} w(\theta) d \theta,
$$

where $w(\theta)$ is the distance between the inner and outer boundaries of the sample, measured along the line that forms 
the angle $\theta$ with the line where the sample is narrowest. We also define the quantities

$$
A_{L}=2 r_{o}^{-2} \int_{r_{i}}^{r_{o}}\left(L r-b_{L} r^{3}\right) \mathcal{R}_{L}^{2} d r
$$

where $r_{i}$ and $r_{o}$ are the inner and outer radii of the unperturbed (symmetric) sample, $b_{L}=\pi H_{L} / \Phi_{0}$,

$$
\begin{gathered}
B_{L}=\int_{r_{i}}^{r_{o}} r \mathcal{R}_{L}^{2} d r \\
C_{L}=\frac{\left(r_{0}-r_{i}\right) r_{o}}{2}\left[\frac{T_{c 0}-T_{L}}{\xi^{2}(0) T_{c 0}}-\left(b_{L} r_{o}-\frac{L+1}{r_{o}}\right)\right. \\
\left.\times\left(b_{L} r_{o}-\frac{L}{r_{o}}\right)\right] \mathcal{R}_{L}\left(r_{o}\right) \mathcal{R}_{L+1}\left(r_{o}\right),
\end{gathered}
$$

where $T_{c 0}$ is the critical temperature at zero magnetic field and $\xi(0)$ is the coherence length at zero temperature,

$$
Q_{L}=\int_{r_{i}}^{r_{o}} r \mathcal{R}_{L}^{4} d r
$$

and

$$
S_{L}=2 \int_{r_{i}}^{r_{o}} r \mathcal{R}_{L}^{2} \mathcal{R}_{L+1}^{2} d r .
$$

We also define $A_{L+1}^{\prime}, B_{L+1}^{\prime}$, and $Q_{L+1}^{\prime}$; these are obtained by the substitution $L \rightarrow L+1$ in the winding number $L$ in Eqs. (3), (4), and (6), while the temperature and the field (and $b_{L}$ ) are left unchanged.

From the perturbation analysis, we obtain that there is a critical point near $\left(H_{L}, T_{L}\right)$, which we denote by $\left(H_{L}^{\text {crit }}, T_{L}^{\text {crit }}\right)$. The critical point is located within the superconducting region. When the passage between the fluxoid states $L$ and $L$ +1 occurs at a temperature above $T_{L}^{\text {crit }}$, it is continuous and mediated by a vortex. If $C_{L}>0$, then the vortex passes through the narrowest part of the sample; if $C_{L}<0$, then it passes through the widest part. It turns out that $C_{L}>0$ for small values of $L$ and becomes negative as $L$ increases; therefore, the vortex passes through the narrow part of the sample for small $L$ and through the wide part for large $L$. The first value of $L$ for which $C_{L}$ is negative depends on the ratio $r_{o} / r_{i}$; the larger this ratio, the smaller the value of $L$ for which $C_{L}$ becomes negative. For temperatures below $T_{L}^{\text {crit }}$, the passage between $L$ and $L+1$ is discontinuous.

To leading term, the position of the critical point is

$T_{L}^{\text {crit }}=T_{L}-\frac{2\left|\beta C_{L}\right| T_{c 0} \xi^{2}(0)\left(Q_{L} Q_{L+1}^{\prime}\right)^{1 / 4}\left(A_{L} \sqrt{Q_{L+1}^{\prime}}-A_{L+1}^{\prime} \sqrt{Q_{L}}\right)}{\left(A_{L} B_{L+1}^{\prime}-A_{L+1}^{\prime} B_{L}\right)\left(\sqrt{Q_{L} Q_{L+1}^{\prime}}-S_{L}\right)}$,

$$
H_{L}^{\text {crit }}=H_{L}+\frac{2\left|\beta C_{L}\right| \Phi_{0}\left(Q_{L} Q_{L+1}^{\prime}\right)^{1 / 4}\left(B_{L} \sqrt{Q_{L+1}^{\prime}}-B_{L+1}^{\prime} \sqrt{Q_{L}}\right)}{\pi r_{o}^{2}\left(A_{L} B_{L+1}^{\prime}-A_{L+1}^{\prime} B_{L}\right)\left(\sqrt{Q_{L} Q_{L+1}^{\prime}}-S_{L}\right)} .
$$

Note that, for simplicity, the definitions of the quantities $\beta, A_{L}, \ldots, S_{L}$ used here are not entirely identical to those in
Ref. 40. Note also that these quantities depend on the normalization of $\mathcal{R}_{L}$, but $T_{L}^{\text {crit }}$ and $H_{L}^{\text {crit }}$ do not.

We point out that for the fluxoid value at which $C_{L}$ changes sign, $C_{L}$ is expected to be small. In view of Eqs. (8) and (9), it follows that for this fluxoid number, the critical point is close to $\left(H_{L}, T_{L}\right)$. As a consequence, for this value of $L$, the vortex-mediated passage occurs only in a particularly small temperature range.

\section{B. Numerical method}

For the numerical method, ${ }^{23}$ we follow the approach of Refs. 2 and 3, and numerically solve the two nonlinear GL equations self-consistently. Here, the demagnetization effect is fully taken into account. Since $d \ll \xi, \lambda$, it is allowed to average the GL equations over the sample thickness. Using dimensionless variables and the London gauge $\operatorname{div} \vec{A}=0$ for the vector potential $\vec{A}$, we write the system of GL equations in the following form:

$$
\begin{gathered}
\left(-i \vec{\nabla}_{2 D}-\vec{A}\right)^{2} \Psi=\Psi\left(1-|\Psi|^{2}\right), \\
-\Delta_{3 D} \vec{A}=\frac{d}{\kappa^{2}} \delta(z) \vec{j}_{2 D},
\end{gathered}
$$

where

$$
\vec{j}_{2 D}=\frac{1}{2 i}\left(\Psi^{*} \vec{\nabla}_{2 D} \Psi-\Psi \vec{\nabla}_{2 D} \Psi^{*}\right)-|\Psi|^{2} \vec{A},
$$

is the density of superconducting current. The superconducting wave function satisfies the boundary conditions $\left(-i \vec{\nabla}_{2 D}\right.$ $-\vec{A})\left.\Psi\right|_{n}=0$ normal to the sample surface and $\vec{A}=\vec{A}_{0}=\frac{1}{2} B r \vec{e}_{\theta}$ far away from the superconductor, where $B$ is the applied field. Here, the distance is measured in units of the coherence length $\xi$, the vector potential in $c \hbar / 2 e \xi$, and the magnetic field in $H_{c 2}=c \hbar / 2 e \xi^{2}=\kappa \sqrt{2} H_{c}$. The superconductor is placed in the $(x, y)$ plane, the external magnetic field is directed along the $z$ axis, and the indices 2D and 3D refer to two- and three-dimensional operators, respectively.

By sweeping up and down the magnetic field, we can find the different (meta-)stable vortex states and their stability range. By comparing the dimensionless Gibbs free energies of the different vortex configurations

$$
F=V^{-1} \int_{V}\left[2\left(\vec{A}-\vec{A}_{0}\right) \cdot \vec{j}_{2 D}-|\Psi|^{4}\right] d \vec{r},
$$

where integration is performed over the sample volume $V$, we find the ground state.

The temperature is indirectly included in $\xi, \lambda$, and $H_{c 2}$, whose temperature dependence is given by

$$
\begin{gathered}
\xi(T)=\frac{\xi(0)}{\sqrt{\left|1-T / T_{c 0}\right|}}, \\
\lambda(T)=\frac{\lambda(0)}{\sqrt{\left|1-T / T_{c 0}\right|}},
\end{gathered}
$$




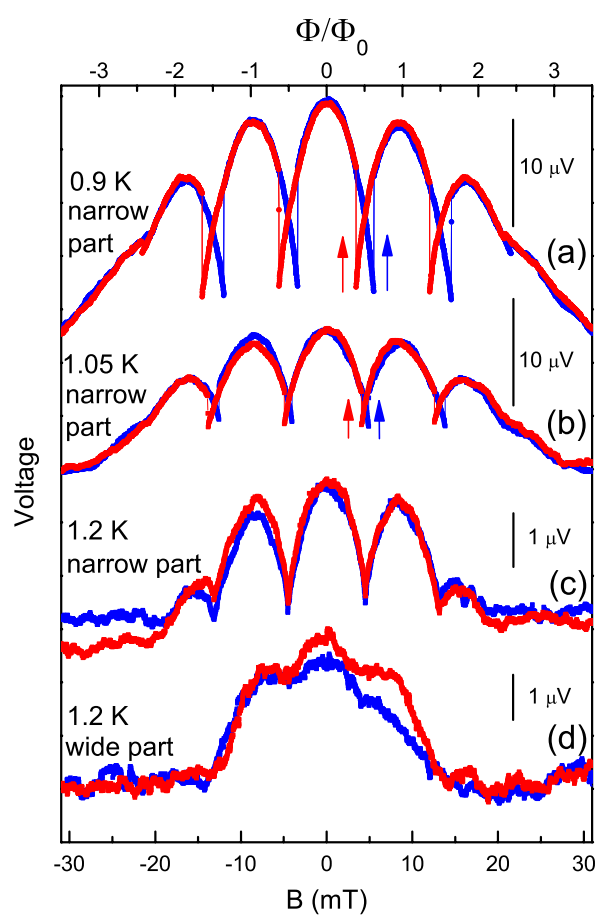

FIG. 2. (Color online) Magnetic field dependence of the junction voltage (current of $0.3 \mathrm{nA}$ is applied) for the junction at the narrowest part of the ring at (a) $0.9 \mathrm{~K}$, (b) $1.05 \mathrm{~K}$, and (c) $1.2 \mathrm{~K}$, and (d) for the junction at the widest part at $1.2 \mathrm{~K}$. The origin and the scale of the ordinate are shifted for clarity. The arrows [blue (red) is for sweep up (down)] indicate the sweep direction of the magnetic field. [(c) and (d)] At $1.2 \mathrm{~K}$, data obtained by sweeping up and down the magnetic field coincide within the accuracy of the measurement. (The error is mainly due to the offset shift of the voltage amplifier.)

$$
H_{c 2}(T)=H_{c 2}(0)\left|1-\frac{T}{T_{c 0}}\right| .
$$

\section{RESULTS}

\section{A. Transitions between fluxoid states}

Figure 2 shows the magnetic field dependence of the junction voltages at a fixed small current of $0.3 \mathrm{nA}$ for increasing and decreasing magnetic fields at several temperatures. The parabola-shaped voltage change is mainly due to a decrease of the energy gap as a consequence of the supercurrent flowing underneath the junction. ${ }^{41}$ Thus, each parabola corresponds to a fluxoid state with a different fluxoid quantum number $L$, and the voltage jumps or kinks between adjacent parabolas are transitions between different fluxoid states. Because the separation between adjacent parabolas is close to $\Phi_{0} / \pi r_{\text {eff }}^{2} \approx 8.85 \mathrm{mT}$, where $r_{\text {eff }}=\sqrt{r_{i} r_{o}}$ is the effective radius of the ring, ${ }^{42} L$ changes by \pm 1 at each transition.

Let us examine the fluxoid-state transitions in more detail. At low temperatures [Fig. 2(a)], clear voltage jumps and hysteresis are seen, which become smaller with increasing temperature [Fig. 2(b)]. As predicted in Refs. 22 and 23, at temperatures close to $T_{c}$, the voltage discontinuities and the

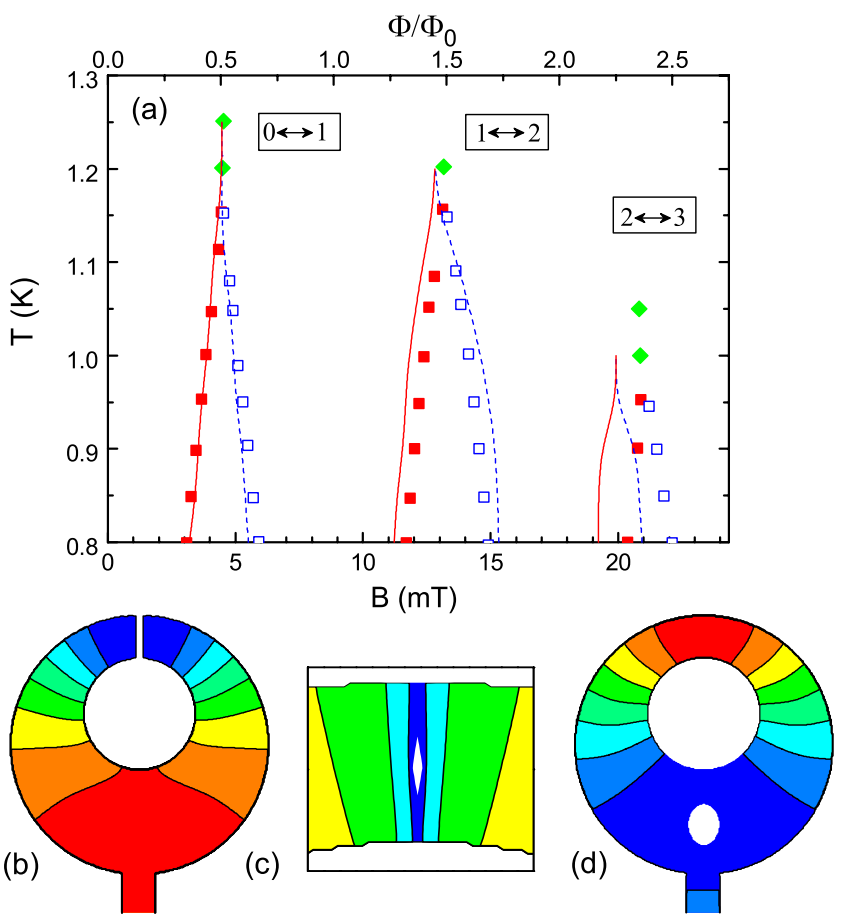

FIG. 3. (Color online) (a) Fluxoid-state transition fields for several temperatures. Open (blue) and closed (red) squares correspond to transition fields for increasing and decreasing magnetic fields, respectively, and the diamonds (green) to the transitions without hysteresis. The solid (red) and dashed (blue) curves correspond to the theoretical expulsion and penetration fields obtained within the framework of the nonlinear Ginzburg-Landau theory. The best agreement with the experimental data was obtained for a system with dimensions $5 \%$ smaller than the experimental sample and for the coherence length $\xi_{\text {num }}(0)=120 \mathrm{~nm}$. Contour plots of the calculated Cooper-pair density in linear scale are shown for the middle of the (b) first and (d) third continuous transitions. The temperatures are 1.2 and $1.0 \mathrm{~K}$, respectively. Red (blue) regions correspond to high (low) Cooper-pair density. At the white region, the Cooper-pair density is less than $10^{-3}$ of the $B=0$ value. (c) A close-up of the narrow part of the ring in (b) but now in logarithmic scale. The white and blue regions correspond to Cooper-pair densities which are less than $10^{-5}$ and $10^{-4}$ of the $B=0$ value, respectively.

hysteresis disappear [Figs. 2(c) and 2(d)], leading to continuous and reversible transitions. Notice that the voltage of the narrowest part at applied magnetic fluxes $\Phi=\pi r_{\mathrm{eff}}^{2} B$ $\approx \pm 0.5 \Phi_{0}$ is close to its normal state value (see the voltage at high magnetic flux, $\left.|\Phi| / \Phi_{0}>2.0\right)$ [Fig. 2(c)], while that of the widest part remains large even at $\Phi= \pm 0.5 \Phi_{0}$ [Fig. 2(d)]. This indicates that the superconductivity in the ring is weakened in a nonuniform manner.

Figure 3 summarizes the magnetic fields where the fluxoid-state transitions occur for increasing (open squares) and decreasing (solid squares) magnetic fields. As temperature increases, the difference between the transition fields in increasing and decreasing magnetic fields becomes smaller, and finally, the transition becomes continuous (diamonds).

In order to get a better understanding of the physics, we compared our experimental results with theoretical calculations done within the framework of the GL theory. The 
dashed and solid curves in Fig. 3 indicate the theoretical transition fields for increasing and decreasing magnetic fields, obtained from the numerical solution of the nonlinear GL equations. When comparing theory with experiment, we found the best agreement, as shown in the figure, when the sizes of the sample were chosen to be $5 \%$ smaller than the experimental sizes and the coherence length $\xi_{\text {num }}(0)$ to be $120 \mathrm{~nm} .{ }^{43}$ At the widest part of the ring, a drain was added, $\xi_{\text {num }}(0)$ wide and $10 \xi_{\text {num }}(0)$ long. This drain influences the theoretical results for the $2 \leftrightarrow 3$ transition and, to a very small extent, also the $1 \leftrightarrow 2$ transition, but not the $0 \leftrightarrow 1$ transition.

By using perturbation theory, ${ }^{40}$ we estimated theoretically the position of the critical points $\left(H_{L}^{\text {crit }}, T_{L}^{\text {crit }}\right)$ : For temperatures below (above) these points, the sample jumps discontinuously (continuously) from one fluxoid state to the next with (without) hysteresis. We obtained the best agreement between this perturbation approach and experiment by increasing $r_{i}$ by $30 \mathrm{~nm}$, decreasing $r_{o}$ by $50 \mathrm{~nm}$, and the coherence length $\xi_{\text {perturb }}(0)=135 \mathrm{~nm} .{ }^{43}$ We found the critical point at $\quad\left(H_{L}^{\text {crit }}(\mathrm{mT}), T_{L}^{\text {crit }}(\mathrm{K})\right)=(4.1,1.17), \quad(12.2, \quad 1.20), \quad$ and $(20.4,0.93)$, for the first three transitions, which are comparable with the measured values $(4.5,1.2),(13.2,1.2)$, and $(20.8,1.0)$.

Thus, both theoretical treatments successfully reproduce the experimental results with appropriate sample sizes as fitting parameters.

These theoretical analyses also predict how the transitions between fluxoid states occur. In agreement with the previous theories, ${ }^{22,23}$ our calculations show that the continuous transitions (without hysteresis) are accompanied by vortices which appear only during the transitions: as the magnetic field is increased (decreased), a vortex appears at the outer (inner) edge of the ring, moves in the radial direction, and finally disappears at the inner (outer) edge.

In the first continuous transition $(L=0 \leftrightarrow 1)$, the vortex passes through the narrowest part of the ring. The calculated Cooper-pair density in the middle of the transition at $1.2 \mathrm{~K}$ is shown in Fig. 3(b) in linear scale. The vortex core where $|\Psi| \approx 0$ (white region) extends across the whole width, and the distribution of the Cooper-pair density around the core is almost uniform in the radial direction, corresponding to the $1 \mathrm{D}$ vortex. On the other hand, for the third continuous transition $(L=2 \leftrightarrow 3)$, a vortex appears at the widest part. Here, the width of the widest part $(0.34 \mu \mathrm{m})$ is comparable to the temperature-dependent coherence length $\xi_{\text {num }}(T=1.2 \mathrm{~K})$ $=\xi_{\text {num }}(0) /\left(1-T / T_{c}\right)^{1 / 2}=0.35 \mu \mathrm{m}$, so that the vortex has a $2 \mathrm{D}$ character, i.e., the Cooper-pair density varies in the radial direction around the core, as shown in Fig. 3(d). In the second transition, the place where the vortex passes sensitively depends on the sizes of the ring. For the best sizes found in the calculations, the vortex enters through the widest part of the ring in the numerical analysis, while it passes through the narrowest part in the perturbation analysis. Nevertheless, in both analyses, it is common that the temperature range for the second continuous transition to occur is very small (about $10 \mathrm{mK}$ ). These features are confirmed experimentally, as described below.

We conclude this section by looking at the Cooper-pair density at the first vortex penetration in more detail. Figure 3(c) shows a contour plot of the calculated Cooper-pair density for the first vortex penetration in logarithmic scale. One can find the 2D distribution of the Cooper-pair density around the center of the vortex. Thus, strictly speaking, i.e., from a mathematical point of view, the Cooper-pair density vanishes only at one point in the narrowest part. However, as shown in the figure, the vortex core is strongly anisotropic and the Cooper-pair density is almost zero $\left(\left|\Psi / \Psi_{0}\right|^{2}\right.$ $<10^{-4}$ ) across the whole width of the narrowest part of the ring $\left(\left|\Psi_{0}\right|^{2}\right.$ is the Cooper-pair density at $\left.B=0\right)$, so that the coherent superconducting order parameter is practically broken at the narrowest part. Therefore, such a state can be called a "quasi-1D" vortex, which is equivalent to the 1D vortex in $1 \mathrm{D}$ rings. ${ }^{23}$ Notice that this is similar to a timedependent phase slip center seen in narrow superconducting wires ${ }^{44}$ with the difference that here it is a stable phase slip center frozen in "time" and "space." This point will be explained in more detail in Sec. V.

\section{B. Temperature dependence of the junction resistances}

The position of the vortex at the continuous transition (i.e., whether the vortex passes through the narrowest or the widest part of the ring) can be inferred from a resistance measurement. Figures 4(a)-4(d) show the temperature dependence of the junction resistance $R$ normalized by its normal state value $R_{n}$ for $B=0$ [Fig. 4(a)] and for magnetic field values close to those of the fluxoid-state transitions without hysteresis [Figs. 4(b)-4(d)]. Generally, as superconductivity develops, the value $R / R_{n}$ increases due to the smaller density of states at low energies and is given by a universal function of the energy gap and the temperature, ${ }^{45}$

$$
\frac{R}{R_{n}}=\left[2 \sum_{m=1}^{\infty}(-1)^{m+1} m \frac{\Delta}{k_{B} T} K_{1}\left(m \frac{\Delta}{k_{B} T}\right)\right]^{-1},
$$

within the BCS theory for $B=0$. Here, $K_{1}$ is the first order modified Bessel function, $\Delta$ the energy gap, $k_{B}$ the Boltzmann constant, and $m$ an integer. This universality is confirmed for our junctions as shown in Fig. 4(a), where both the ratio for the narrowest part, $\left(R / R_{n}\right)_{N}$, and that for the widest part, $\left(R / R_{n}\right)_{W}$, have the same temperature dependence and are in good agreement with Eq. (15) (solid curve) at temperatures close to $T_{c} \cdot{ }^{46}$ On the other hand, for the first transition [Fig. 4(b)], $\left(R / R_{n}\right)_{N}$ stays close to 1 between $1.2 \mathrm{~K}$ and $T_{c}(4.5 \mathrm{mT})=1.32 \mathrm{~K}$, while $\left(R / R_{n}\right)_{W}$ increases as temperature decreases below $T_{c}(4.5 \mathrm{mT})$. The temperature range where the increase in $\left(R / R_{n}\right)_{N}$ is suppressed agrees well with the range where the transitions without hysteresis is observed ( $T \geqslant 1.2 \mathrm{~K}$, see Fig. 3), strongly indicating that a stable vortex exists in the narrowest part of the ring. Similarly, for the third transition [Fig. 4(d)], the increase in $\left(R / R_{n}\right)_{W}$ is suppressed in comparison with $\left(R / R_{n}\right)_{N}$ in a wide temperature range, indicating that a vortex is situated in the widest part of the ring. On the other hand, for the second transition [Fig. 4(c)], the difference between $\left(R / R_{n}\right)_{N}$ and $\left(R / R_{n}\right)_{W}$ is rather small, corresponding to a narrow temperature range for the existence of the vortex, which is derived in the theoretical analyses described above. Thus, the position of the vortex agrees well with the theoretical results. 

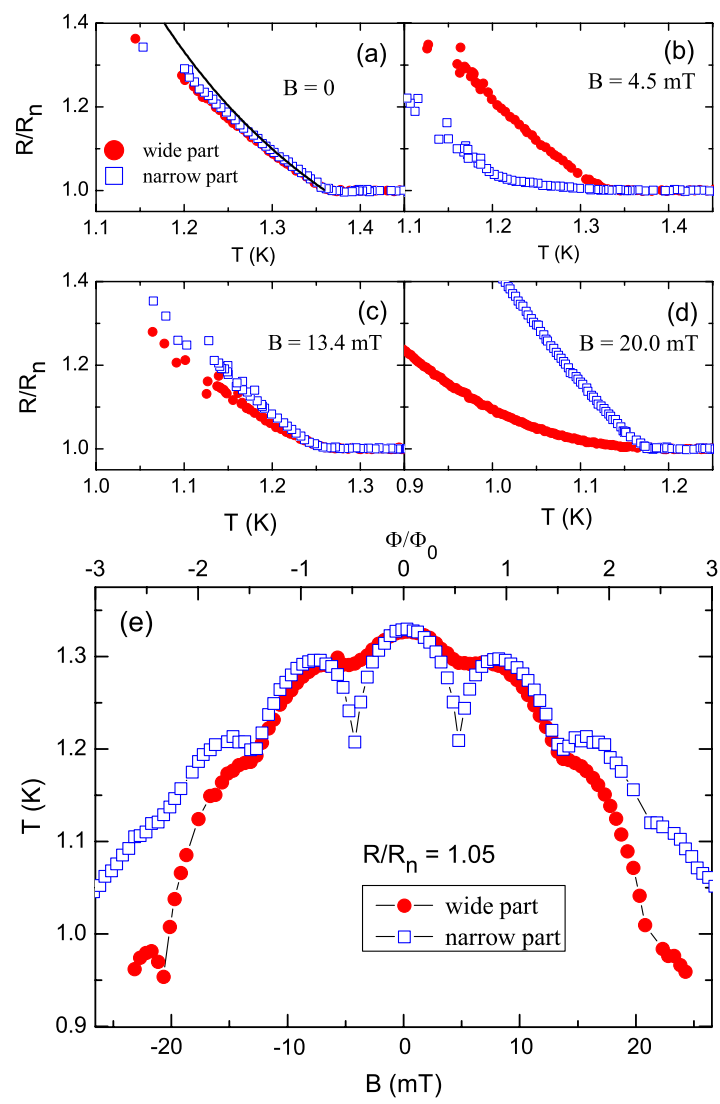

FIG. 4. (Color online) $[(a)-(d)]$ Temperature dependence of the zero-bias junction resistance $R$ normalized by its normal state value $R_{n}$ for junctions attached to the widest part [closed (red) circle] and the narrowest part [open (blue) square] of the ring for applied magnetic fields of (a) 0, (b) $4.5 \mathrm{mT}$, (c) $13.4 \mathrm{mT}$, and (d) $20.0 \mathrm{mT}$. The magnetic field values for (b)-(d) are close to those of the fluxoidstate transitions without hysteresis. The resistance was obtained from a linear fit of the $I-V$ curve at $|V|<50 \mu \mathrm{V}$. In (a), the theoretical $R / R_{n}$ ratio [Eq. (15)] is also shown (solid curve). (e) Temperature where the resistance ratio $R / R_{n}$ reaches 1.05 is plotted as a function of the applied magnetic field.

We repeated similar resistance measurements for many magnetic field values with a step of $0.5 \mathrm{mT}$, and obtained Fig. 4(e), which shows the temperature where $R / R_{n}$ becomes 1.05 as a function of magnetic field. A steep decrease of the temperature around $\Phi / \Phi_{0} \approx \pm 0.5$ for the narrowest part confirms the penetration of a vortex, but the width of the dip $(\approx 2.5 \mathrm{mT})$ is still larger than the theoretical field region for the existence of the vortex core $(\approx 0.3 \mathrm{mT})$, indicating that the Cooper-pair density starts decreasing as a precursor of the vortex penetration. For the third transition, this precursor is also seen, but now for the widest part of the ring.

\section{DISCUSSIONS}

We emphasize that the quasi-1D vortex we observed at the continuous transitions is not due to a thermally activated process. The present vortex does not move when the magnetic field is kept fixed, i.e., the vortex is frozen in time and space. The close agreement between our theoretical simulations and the experimental results shows clearly that the frozen vortex state is the ground state. Experimentally, this is proven in Figs. 2(c) and 4(b), where the superconductivity at the narrowest part is suppressed in the temperature range where the hysteresis disappears. Note that during a thermally activated transition, the order parameter would remain finite everywhere in the ring, ${ }^{47}$ while in our case, it vanishes at some place along the ring.

It is rather easy to show that the influence of thermally activated processes is vanishingly small. The ratio $E_{a} / k_{B} T$ is about $10^{3}$ at $T=1.2 \mathrm{~K}\left[E_{a} \sim V H_{c}^{2} / 8 \pi\right.$ is the condensation energy in the narrowest part of the ring with length $\xi(T=1.2 \mathrm{~K}) \sim 0.43 \mu \mathrm{m}$, width $w=0.14 \mu \mathrm{m}$, and volume $V$ $\sim w d \xi=0.0018 \mu \mathrm{m}^{3}$ in zero magnetic field], and hence, the probability of the thermofluctuations $\sim \exp \left(-E_{a} / k_{B} T\right)$ is negligible at this temperature even if the order parameter is considerably suppressed at $\Phi=\Phi_{0} / 2$, which gives $E_{a} / k_{B} T$ $\sim 10^{2}$.

Besides, the experimentally observed vortex state is completely different from what is seen in transitions accompanied by hysteresis. Actually, an earlier theoretical study ${ }^{31}$ showed that a transient state with a vortex sitting somewhere along the superconducting ring is never stabilized when hysteresis is present, and our theoretical simulation shows that this is the case for the present ring. This is also experimentally confirmed in, say, Fig. 2(a), where the junction voltage at the transition is larger than the normal state value, showing that the narrowest part of the ring does not contain a normal vortex core as the transient state. Notice that this is not the case when the hysteresis disappears in Fig. 2(c), where the junction voltage at the transition is the same as the normal state value seen at high magnetic fields.

As described in Sec. IV, the position of the vortex at a continuous transition depends on the magnetic flux. The reason why the vortex passes through the narrowest part of the ring in the first continuous transition $(L=0 \leftrightarrow 1)$ can be explained in the following way: At $L=0$ under magnetic field, a shielding supercurrent flows along the outer boundary of the ring. Its nominal decay length, or the effective penetration depth, ${ }^{44}$ is about $1 \mu \mathrm{m}$, which is comparable to the sample size. Thus, the supercurrent is distributed over the ring. Because of current conservation, the current density is maximal in the narrowest place, and hence, the order parameter is minimal there, resulting in vortex penetration at the narrowest place. For $L \neq 0$, the current from the magnetic flux trapped in the hole sets in. This current flows along the inner boundary of the ring and its direction is opposite to the direction of the shielding supercurrent, so that it is possible that the current compensation leads to the vortex penetration at the widest part of the ring. This is the simplest explanation of the phenomenon. The existence of the thin Al drain lead attached to the widest part of our sample does not cause a serious change in this discussion; the sample shape and the spatial current distribution still have mirror symmetry, so that the position of the vortex penetration is limited to the narrowest and the widest part of the ring.

Here, we comment on the effect of the finite junction area. In Fig. 5, we calculated the average of the order parameter over the junction area, $|\Psi|_{\text {average }}$, for the magnetic field of the 


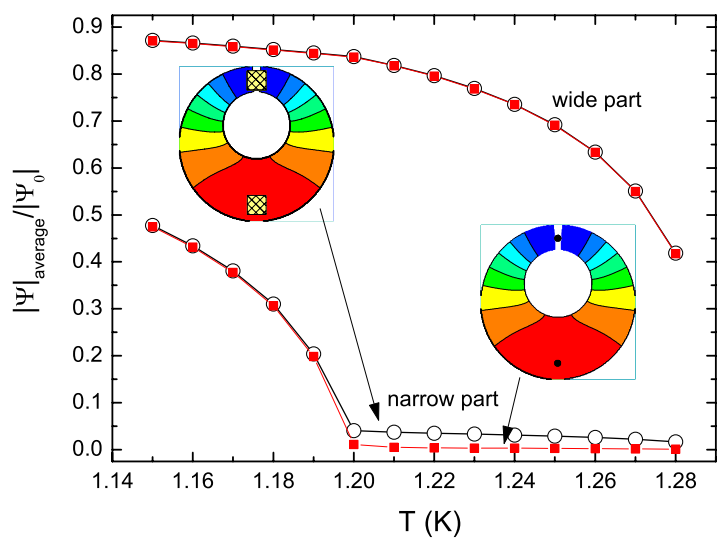

FIG. 5. (Color online) The calculated average of the order parameter over the junction area for the magnetic field of the first continuous transition. The junctions are placed at the center of the narrowest part of the ring and at a distance of $0.09 \mu \mathrm{m}$ from the outer boundary of the widest part. Squares indicate the results for infinitesimal junction area, and circles for the junction area of 0.1 $\times 0.1 \mu \mathrm{m}^{2}$. The insets show the Cooper-pair density at $1.2 \mathrm{~K}$. The hatched squares in the left inset and the dots in the right inset indicate the junctions.

first continuous transition. We assume that one junction is placed at the center of the narrowest part of the ring, and the other at a distance of $0.09 \mu \mathrm{m}$ from the outer boundary of the widest part. For a junction with infinitesimal area, $|\Psi|_{\text {average }}$ stays almost zero at the narrowest part above $1.2 \mathrm{~K}$ (squares), while for a finite junction area $\left(0.1 \times 0.1 \mu \mathrm{m}^{2}\right)$, $|\Psi|_{\text {average }}$ slightly increases while the temperature decreases to $1.2 \mathrm{~K}$. This means that even if a vortex is situated at the narrowest part of the ring, the probed Cooper-pair density (or measured junction resistance) may be slightly different from the normal state value due to the finite junction area, and shows a temperature dependence. Thus, the slight change of $\left(R / R_{n}\right)_{N}$ between $1.2 \mathrm{~K}$ and $T_{c}(4.5 \mathrm{mT})=1.32 \mathrm{~K}$ in Fig. $4(\mathrm{~b})$ is attributed to the effect of the finite width of the junction area.

Finally, we comment on the possible existence of the quasi-1D vortex in a previous experiment. Liu et al. ${ }^{36}$ observed a finite resistance at $\pm 0.5 \Phi_{0}$ well below $T_{c}$ in a thin hollow $\mathrm{Al}$ cylinder, which was explained by the global destruction of superconductivity due to the Little-Parks effect. We argue that the result might also arise from the presence of a 1D vortex due to small variations in the sample width, which readily explains the observed decrease in the sample resistance in comparison with the normal state value.

\section{CONCLUSION}

We investigated transitions between different fluxoid states in a mesoscopic superconducting ring with an offcentered hole, emphasizing the existence of a different type of vortex that results in a continuous fluxoid transition. Experimentally, we used the MSTJ method to compare the strength of superconductivity at the narrowest and the widest part of the ring. The experimental results are corroborated by a theoretical analysis within the framework of the nonlinear Ginzburg-Landau theory. We observed reversible and continuous transitions between adjacent fluxoid states at temperatures close to the onset of superconductivity. Our theoretical simulations reproduce closely the experimental transition fields with reasonable values for the fitting parameters (i.e., the sizes of the ring and the coherence length). Besides, it predicted the existence of a different type of vortex at the continuous transitions: as the magnetic field is increased (decreased), the vortex appears at the outer (inner) boundary of the ring, moves in the radial direction, and finally disappears at the inner (outer) boundary. This vortex passes through the narrowest part of the ring at low magnetic fields and through the widest part of the ring at high magnetic fields, because of the interaction between the shielding supercurrent and the current around the flux in the hole. In particular, when the vortex is situated at the narrowest part, its core becomes highly anisotropic and the Cooper-pair density is almost zero across the whole width. Thus, the vortex is analogous to a $1 \mathrm{D}$ vortex in the 1D limit, and can be called a quasi- $1 D$ vortex. Experimentally, by comparing the temperature dependences of the junction resistance at the narrowest and the widest part of the ring, we confirmed the existence of the vortex at the continuous fluxoid-state transitions. We determined the position of the vortex, which agrees with our theoretical predictions. These results confirm the existence of a different type of vortex at the continuous fluxoid-state transitions.

\section{ACKNOWLEDGMENTS}

This work was supported by the University of Tsukuba Nanoscience Special Project, Grant-in-Aid for Scientific Research (B) (17340101), CTC-NES Program of JSPS, the Flemish Science Foundation (FWO-Vl), the Belgian Science Policy (IAP), and the ESF-AQDJJ program. D.Y.V. acknowledges support from the Dynasty foundation. B.J.B. acknowledges support from FWO-Vl. J.B. acknowledges support from the Israel Science Foundation (4/03-11.7).
${ }^{1}$ V. V. Moshchalkov, X. G. Qiu, and V. Bruyndoncx, Phys. Rev. B 55, 11793 (1997).

${ }^{2}$ V. A. Schweigert and F. M. Peeters, Phys. Rev. B 57, 13817 (1998)

${ }^{3}$ V. A. Schweigert, F. M. Peeters, and P. S. Deo, Phys. Rev. Lett. 81, 2783 (1998).
${ }^{4}$ J. J. Palacios, Phys. Rev. B 58, R5948 (1998).

${ }^{5}$ G. M. Braverman, S. A. Gredeskul, and Y. Avishai, Phys. Rev. B 59, 12039 (1999).

${ }^{6}$ J. J. Palacios, Phys. Rev. Lett. 84, 1796 (2000).

${ }^{7}$ V. Hakim, A. Lemaître, and K. Mallick, Phys. Rev. B 64, 134512 (2001). 
${ }^{8}$ B. J. Baelus, L. R. E. Cabral, and F. M. Peeters, Phys. Rev. B 69, 064506 (2004).

${ }^{9}$ L. R. E. Cabral, B. J. Baelus, and F. M. Peeters, Phys. Rev. B 70, 144523 (2004).

${ }^{10}$ L. F. Chibotaru, A. Ceulemans, V. Bruyndoncx, and V. V. Moshchalkov, Nature (London) 408, 833 (2000).

${ }^{11}$ L. F. Chibotaru, A. Ceulemans, V. Bruyndoncx, and V. V. Moshchalkov, Phys. Rev. Lett. 86, 1323 (2001).

${ }^{12}$ V. R. Misko, V. M. Fomin, J. T. Devreese, and V. V. Moshchalkov, Phys. Rev. Lett. 90, 147003 (2003).

${ }^{13}$ L. F. Chibotaru, G. Teniers, A. Ceulemans, and V. V. Moshchalkov, Phys. Rev. B 70, 094505 (2004).

${ }^{14}$ R. Geurts, M. V. Milosević, and F. M. Peeters, Phys. Rev. Lett. 97, 137002 (2006).

${ }^{15}$ A. K. Geim, I. V. Grigorieva, S. V. Dubonos, J. G. S. Lok, J. C. Maan, A. E. Filippov, and F. M. Peeters, Nature (London) 390, 256 (1997).

${ }^{16}$ V. Bruyndoncx, J. G. Rodrigo, T. Puig, L. Van Look, V. V. Moshchalkov, and R. Jonckheere, Phys. Rev. B 60, 4285 (1999).

${ }^{17}$ A. K. Geim, S. V. Dubonos, J. J. Palacios, I. V. Grigorieva, M. Henini, and J. J. Schermer, Phys. Rev. Lett. 85, 1528 (2000).

${ }^{18}$ A. Kanda, B. J. Baelus, F. M. Peeters, K. Kadowaki, and Y. Ootuka, Phys. Rev. Lett. 93, 257002 (2004).

${ }^{19}$ B. J. Baelus, A. Kanda, F. M. Peeters, Y. Ootuka, and K. Kadowaki, Phys. Rev. B 71, 140502(R) (2005).

${ }^{20}$ I. V. Grigorieva, W. Escoffier, J. Richardson, L. Y. Vinnikov, S. Dubonos, and V. Oboznov, Phys. Rev. Lett. 96, 077005 (2006).

${ }^{21}$ B. J. Baelus, A. Kanda, N. Shimizu, K. Tadano, Y. Ootuka, K. Kadowaki, and F. M. Peeters, Phys. Rev. B 73, 024514 (2006).

${ }^{22}$ J. Berger and J. Rubinstein, Phys. Rev. B 59, 8896 (1999).

${ }^{23}$ D. Y. Vodolazov, B. J. Baelus, and F. M. Peeters, Phys. Rev. B 66, 054531 (2002).

${ }^{24}$ P.-G. de Gennes, C. R. Seances Acad. Sci., Ser. 1 292, 279 (1981).

${ }^{25}$ J. P. Straley and P. B. Visscher, Phys. Rev. B 26, 4922 (1982).

${ }^{26}$ H. J. Fink, A. López, and R. Maynard, Phys. Rev. B 26, 5237 (1982); H. J. Fink, V. Grŭnfeld, and A. López, ibid. 35, 35 (1987); H. J. Fink, J. Loo, and S. M. Roberts, ibid. 37, 5050 (1988).

${ }^{27}$ J. Berger and J. Rubinstein, Phys. Rev. Lett. 75, 320 (1995).

${ }^{28}$ E. M. Horane, J. I. Castro, G. C. Buscaglia, and A. López, Phys.
Rev. B 53, 9296 (1996).

${ }^{29}$ J. Berger and J. Rubinstein, Phys. Rev. B 56, 5124 (1997).

${ }^{30}$ J. I. Castro and A. López, Phys. Rev. B 72, 224507 (2005).

${ }^{31}$ B. J. Baelus, F. M. Peeters, and V. A. Schweigert, Phys. Rev. B 61, 9734 (2000).

${ }^{32}$ X. Zhang and J. C. Price, Phys. Rev. B 55, 3128 (1997).

${ }^{33}$ D. Davidović, S. Kumar, D. H. Reich, J. Siegel, S. B. Field, R. C. Tiberio, R. Hey, and K. Ploog, Phys. Rev. B 55, 6518 (1997).

${ }^{34}$ A. Kanda, M. C. Geisler, K. Ishibashi, Y. Aoyagi, and T. Sugano, Microelectron. Eng. 47, 389 (1999).

${ }^{35}$ S. Pedersen, G. R. Kofod, J. C. Hollingbery, C. B. Sorensen, and P. E. Lindelof, Phys. Rev. B 64, 104522 (2001).

${ }^{36}$ Y. Liu, Yu. Zadorozhny, M. M. Rosario, B. Y. Rock, P. T. Carrigan, and H. Wang, Science 294, 2332 (2001).

${ }^{37}$ D. Y. Vodolazov, F. M. Peeters, S. V. Dubonos, and A. K. Geim, Phys. Rev. B 67, 054506 (2003).

${ }^{38}$ M. Morelle, D. S. Golubović, and V. V. Moshchalkov, Phys. Rev. B 70, 144528 (2004).

${ }^{39}$ R. Benoist and W. Zwerger, Z. Phys. B: Condens. Matter 103, 377 (1997); V. V. Moshchalkov, V. Bruyndoncx, and L. Van Look, in Connectivity and Superconductivity, edited by J. Berger and J. Rubinstein (Springer, Berlin, 2000).

${ }^{40} \mathrm{~J}$. Berger, in Connectivity and Superconductivity, edited by J. Berger and J. Rubinstein (Springer, Berlin, 2000).

${ }^{41}$ A. Kanda and Y. Ootuka, Physica C 404, 205 (2004).

${ }^{42}$ R. M. Arutunian and G. F. Zharkov, J. Low Temp. Phys. 52, 409 (1983).

${ }^{43}$ The difference of the fitted sample sizes from the actual values probably comes from the boundary roughness and the surface oxide layer. A similar effect has been seen previously for the thickness of Al films [see T. Wada, S. Haraichi, K. Ishii, H. Hiroshima, M. Komuro, and S. M. Gorwadkar, J. Vac. Sci. Technol. A 16, 1430 (1998)].

${ }^{44}$ M. Tinkham, Introduction to Superconductivity, 2nd ed. (McGraw-Hill, New York, 1996).

${ }^{45}$ I. Giaever and K. Megerle, Phys. Rev. 122, 1101 (1961).

${ }^{46}$ The deviation of the experimental data from Eq. (15) at low temperatures is due to the nonlinear $I-V$ characteristics at $|V|$ $<50 \mu \mathrm{V}$, where the linear fit was performed.

${ }^{47}$ K. A. Matveev, A. I. Larkin, and L. I. Glazman, Phys. Rev. Lett. 89, 096802 (2002). 